\title{
Conservación de Mango Mínimamente Procesado usando un Recubrimiento Comestible a base de Aceite de Aguacate
}

\author{
Saúl Dussán-Sarria*, Jorge I. Ramírez-Yela y Jose I. Hleap-Zapata \\ Fac. de Ingeniería, Depto. Ingeniería y Administración, Univ. Nacional de Colombia. Sede Palmira. A.A. \\ 237. Palmira, Valle del Cauca-Colombia. (e-mail: sdussan@unal.edu.co).
}

${ }^{*}$ Autor a quien debe ser dirigida la correspondencia.

Recibido Oct. 5, 2016; Aceptado Dic. 1, 2016; Versión final Feb. 28, 2016, Publicado Jun. 2017

\begin{abstract}
Resumen
El objetivo de este trabajo fue evaluar el efecto de la aplicación de diferentes recubrimientos comestibles preparados con aceite virgen de aguacate, aplicados a mango variedad Tommy Atkins mínimamente procesado, almacenado a dos temperaturas, $5 \pm 2^{\circ} \mathrm{C}$ y $15 \pm 2^{\circ} \mathrm{C}$, con humedad relativa de $80 \pm 2 \%$. Los frutos fueron seleccionados homogéneamente con un índice de madurez 3 , se lavaron, pelaron y cortaron en trozos. El mango mínimamente procesado fue tratado previamente con ácidos orgánicos a $1 \% \mathrm{v} / \mathrm{v} \mathrm{y} \mathrm{CaCl}_{2}$ a $1 \% \mathrm{v} / \mathrm{v}$. Se evaluó la calidad del producto cada 4 días hasta el día 16 a través de sus atributos físicoquímicos, sensoriales y microbiológicos para finalmente definir la condición más adecuada de conservación. El recubrimiento F1 (cera carnauba $0,78 \%$, glicerol 2,64\%, aceite de aguacate 1,20\%, acido esteárico $1,54 \%$, almidón de yuca $2,39 \%$, goma xanthan $0,01 \%$ y agua $91,44 \%$ ) bajo refrigeración a $5 \pm 2^{\circ} \mathrm{C}$ y $80 \pm 2 \%$ de humedad relativa proporcionó las mejores propiedades organolépticas del fruto.
\end{abstract}

Palabras clave: Mangifera indica. L; Tommy Atkins; conservación; fruta; aceite virgen.

\section{Conservation of Fresh-Cut Mango using an Edible Coating based on Avocado Oil}

\begin{abstract}
The objective of this study was to evaluate the effect of the application of different edible coatings prepared with virgin avocado oil, applied to fresh-cut Tommy Atkins mango stored at two temperatures, $5 \pm 2^{\circ} \mathrm{C}$ and $15 \pm 2^{\circ} \mathrm{C}$, with $80 \pm 2 \%$ relative humidity. The fruits were homogeneously selected with maturity index 3 , were washed, peeled and cut into pieces. The slices of mango were pretreated with organic acids at $1 \% \mathrm{v} / \mathrm{v}$ and $\mathrm{CaCl}_{2}$ at $1 \% \mathrm{v} / \mathrm{v}$. The quality of the product was evaluated every 4 days until day 16 determining its physical-chemical, microbiological and sensory attributes, to finally define the most appropriate storing conditions. The coating F1 (carnauba Wax $0.78 \%$, glycerol $2.64 \%$, avocado oil $1.20 \%$, stearic acid $1.54 \%$, cassava starch $2.39 \%$, xanthan gum $0.01 \%$ and water $91,44 \%$ ) under refrigeration at $5 \pm 2^{\circ} \mathrm{C}$ and $80 \pm 2 \%$ relative humidity provided the best organoleptic properties of the fruit.
\end{abstract}

Keywords: Mangifera indica. L; Tommy Atkins; conservation; fruit; virgin oil. 


\section{INTRODUCCIÓN}

El mango (Mangifera indica. L.) es considerado uno de los principales productos frutícolas de origen tropical ampliamente cultivado en los trópicos y subtrópico, siendo India, China, Tailandia, Indonesia, Filipinas, Pakistán y México los principales países productores (Tharanathan et al., 2006). El mango es una de las frutas tropicales más importantes en el mundo, principalmente por su agradable sabor, aroma y por su alto valor nutricional, es un fruto rico en agua, azúcares, fibra, minerales y vitaminas, (Singh et al., 2013). La comercialización de esta fruta se lleva a cabo en un período muy corto, pues es un producto perecedero y susceptible al deterioro causado por desórdenes fisiológicos y patológicos en la poscosecha (Cáceres et al. 2003). Entre los tratamientos pososecha complementarios en frutas se encuentran los recubrimientos comestibles céreos a base de resinas como alternativa de mejora de apariencia y prolongamiento de la vida comercial por reducción de la pérdida de peso (Báez et al., 2000).

Los alimentos inocuos y además convenientes debido a los estilos modernos de vida y hábitos de consumo son una tendencia mundial actual. La International Fresh-cut Produce Association (IFPA, 2004), define a los productos mínimamente procesados como "cualquier fruta u hortaliza, o combinación de estas, que haya sido físicamente alterada, pero que permanece en estado fresco". El mango mínimamente procesado refrigerado es una buena opción en el mercado; y responde a la tendencia de satisfacer la necesidad del mundo moderno que dispone de menos tiempo para la preparación de sus alimentos; en tanto que su grado de interés por comidas saludables libres de conservantes y otros aditivos químicos es cada vez más alto (Laurila, 1998). El mango cortado es muy perecedero debido a que pierde la protección del pericarpio y son modificadas sus propiedades fisicoquímicas por los trastornos metabólicos que se producen por el corte (Kader, 2008), sumado a esto, este tipo de procesamiento genera oscurecimiento enzimático y disminución de la firmeza (Chiumarelli et al., 2011).

De acuerdo a Barbagallo et al. (2009), la intensidad del oscurecimiento enzimático en vegetales cortados depende de la actividad oxidativa relativa de las enzimas polifenoloxidasa y pectinametilesterasa, la concentración de sustrato (compuestos fenólicos) y del oxígeno molecular junto con apropiadas condiciones de $\mathrm{pH}$, temperatura y actividad de agua del vegetal. Del manejo de estos factores resulta también el control de este tipo de oxidación. En la revisión de literatura se encuentra que los almidones de interés como el derivado de yuca (Manihot esculenta) plastificado con glicerol (Estrada-Mesa et al., 2015), incorporados con compuestos antimicrobianos naturales (Kechichian et al., 2010), y almidón de maíz estándar y pregelatinizado hacen parte de los biopolímeros de interés por su bajo precio y accesibilidad (Liu et al., 2009). Los polisacáridos y las proteínas son buenos materiales para la formación de recubrimientos comestibles, ya que muestran excelentes propiedades mecánicas y estructurales, pero presentan una pobre capacidad de barrera frente a la humedad. Este problema no se encuentra en los lípidos dadas sus propiedades hidrofóbicas, especialmente los que poseen puntos de fusión altos tales como la cera de abejas y la cera carnauba (Pérez-Gago y Jong-Whan, 2014).

Diferentes tratamientos poscosecha como los recubrimientos comestibles y la aplicación o no de calor, han sido investigados con el fin de mantener las propiedades del mango fresco cortado por más tiempo (Souza et al., 2006; Sothornvit y Rodsamran, 2008; Djioua et al., 2009; Djioua et al., 2010; Chiumarelli et al., 2011; Dussán-Sarria et al., 2014). En este sentido, el objetivo de este trabajo fue evaluar la calidad durante la conservación de mango variedad Tommy atkins mínimamente procesado con el uso agroindustrial de recubrimientos comestibles preparados con aceite de aguacate.

\section{MATERIALES Y MÉTODOS}

El experimento se realizó en el laboratorio de frutas y hortalizas de la Universidad Nacional de Colombia (Palmira, Valle del Cauca, Colombia). Fueron utilizados mangos variedad 'Tommy Atkins' seleccionados y clasificados por tamaño uniforme y estado de madurez 3 según Kader (2008). Los frutos presentaban condiciones de color, firmeza interna y atributos de calidad adecuados para el procesamiento mínimo en fresco como lo describen Djioua et al. (2009) y Chien et al. ( 2007).

Los frutos fueron mantenidos bajo refrigeración $\left(10^{\circ} \mathrm{C}\right)$ un día antes de ser sometidos al procesamiento con el fin de evitar cambios acelerados en su metabolismo. Al día siguiente durante el procesamiento se retiró la cáscara de los frutos con cuchillos en acero inoxidable desinfectados, y a través de ensayos preliminares evaluando rodajas, cubos y trozos longitudinales. Los trozos de $6 \mathrm{~cm} \times 2 \mathrm{~cm} \times 2 \mathrm{~cm}$ proporcionaron el mejor rendimiento del $68,17 \%$ en la relación pulpa/residuos (Dussán-Sarria et al., 2014). Posteriormente los trozos de mango se sumergieron en hipoclorito de sodio a 10 ppm durante 1 minuto, se dejaron escurrir por 2 min para posteriormente ser sumergidos en una solución de ácido cítrico al 1\% (v/v) + ácido ascórbico al 1\% $(\mathrm{v} / \mathrm{v})+1 \%(\mathrm{v} / \mathrm{v}) \mathrm{CaCl}_{2}$ durante 3 minutos. 
Fueron evaluadas dos formulaciones de recubrimiento comestible donde las proporciones utilizadas de sus componentes se observan en la Tabla 1 y en la cual se siguió el procedimiento de formulación indicado por Dussán- Sarria et al. (2014) con la variación de la utilización de aceite de aguacate en la emulsión como barrera al vapor de agua en lugar del aceite de carnauba y canola, además se incorporó goma xantano para evitar la separación de fases. Las proporciones en porcentaje se definieron según experimentación previa con análisis sensorial afectivo de apariencia. El ácido esteárico se utilizó para reducir el punto de fusión de la cera de carnauba y la goma xantano es un polímero y se utilizó como estabilizante de las emulsiones.

Tabla 1: Proporciones de los componentes del recubrimiento comestible. F1: Formulación 1; F2: Formulación 2.

\begin{tabular}{lll}
\hline Componente & F1 (\%) & F2 (\%) \\
\hline Cera carnauba & 0,78 & 0,40 \\
Glicerol & 2,64 & 2,62 \\
Aceite de Aguacate & 1,20 & 2,08 \\
Acido esteárico & 1,54 & 0,61 \\
Almidón de yuca & 2,39 & 2,60 \\
Goma Xantano & 0,01 & 0,13 \\
Agua & 91,44 & 91,57 \\
Total & 100 & 100 \\
\hline
\end{tabular}

Los trozos de mango mínimamente procesado fueron sumergidos durante 2 minutos en el recubrimiento comestible formado y posteriormente fueron secados con aire forzado con ayuda de un ventilador y empacados, estos procedimientos se realizaron a temperatura ambiente $\left(\approx 28^{\circ} \mathrm{C}\right)$. Como empaque fue utilizada la caja PET (polietileno tereftalato) de medidas $12 \mathrm{~cm} \times 8 \mathrm{~cm} \times 4 \mathrm{~cm}$ y las siguientes permeabilidades: al $\mathrm{CO}_{2}$ de $112,3 \mathrm{~cm}^{3} \mathrm{~m}^{-2}$ día $\mathrm{a}^{-1}$ y al $\mathrm{O}_{2}$ de $3945,2929 \mathrm{~cm}^{3} \mathrm{~m}^{-2}$ día ${ }^{-1}$. Fueron acondicionados $150 \mathrm{~g}$ de trozos de mango por empaque y almacenado a dos temperaturas y manteniendo elevada la humedad relativa del aire a $80 \pm 2 \%$. La temperatura de almacenamiento a $5 \pm 2^{\circ} \mathrm{C}$ se utilizó debido a las varias recomendaciones observadas en la literatura para vegetales mínimamente procesados (Sothornvit y Rodsamran, 2008; Kader, 2008) y la temperatura de almacenamiento a $15 \pm 2^{\circ} \mathrm{C}$ se utilizó para simular las condiciones reales de las góndolas o estantes en supermercado local. Los trozos de mango fueron evaluados a los $0,4,8,12,16$ días de almacenamiento. La evaluación de atributos se realizó en cuatro aspectos: análisis sensorial, parámetros de color, resistencia a la penetración y análisis microbiológico

\section{Análisis sensorial}

A través de 30 jurados no entrenados se evaluaron sensorialmente los cambios en la conjugación sensorial de apariencia y sabor del mango en trozos. El ensayo afectivo siguió la escala no estructurada tipo lineal de $10 \mathrm{~cm}$ siendo definida por expresiones cualitativas en las extremidades: no me gustó extremamente (1) y me gustó extremamente (10), siendo la nota de 5 la mínima aceptable sensorialmente en este estudio (Anzaldúa-Morales, 1994).

\section{Parámetros de color}

El color de la superficie de los trozos de mango se midió utilizando un colorímetro Marca Konica Minolta, Modelo: $C R$ - 400, escala $\left(L^{*}, a^{*}, b^{*}\right)$, D65 como fuente luminosa y $10^{\circ}$ ángulo de observación, Las mediciones de color se expresan en términos de luminosidad $L^{*}\left(L^{*}=0\right.$ para el negro y $L^{*}=100$ para el blanco), y los parámetros de cromaticidad $a^{*}$ (verde [-], rojo [+]) y $b^{*}$ (azul[-], amarillo [+]). A partir de estos parámetros, fueron calculadas las coordenadas $\mathrm{C}^{*}$ (croma) y $\mathrm{H}^{*}$ (tono) utilizando la ecuación 1 y la ecuación 2, respectivamente (Chiumarelli et al., 2011).

$$
\begin{aligned}
& C=\left(a^{2}+b^{2}\right)^{1 / 2} \\
& H=\operatorname{artan}\left(\frac{b}{a}\right)
\end{aligned}
$$

\section{Resistencia a la penetración}

La fuerza a la penetración medida en los trozos de mango se determinó manualmente con ayuda del penetrómetro FORCE DIAL FDK 30 con capacidad máxima de $14 \mathrm{kgf}$. Se utilizó una punta de $8 \mathrm{~mm}$ de diámetro y la fuerza se aplicó en dirección perpendicular a la superficie de la muestra de mango, los valores se expresan en Kgf (Djioua et al., 2009). 


\section{Análisis Microbiológico}

Se realizó análisis microbiológico a los trozos de mango en todos los tratamientos al inicio y al final de la experimentación, teniendo en cuenta presencia de coliformes totales y fecales, salmonella en $25 \mathrm{~g}$, mohos y levaduras. Las pruebas microbiológicas se realizaron en el laboratorio Bioindustrial Ángel en Cali-Colombia.

\section{Análisis experimental y estadístico}

Fueron evaluados 6 tratamientos derivados de 2 condiciones de temperatura y 3 condiciones de recubrimiento comestible designados de la forma que se muestra en la Tabla 2 . Se utilizó el delineamiento experimental completamente al azar por triplicado de cada tratamiento, la unidad experimental fue cada envase conteniendo el fruto cortado y se aplicó análisis estadístico basado en estadística descriptiva, análisis de varianza y comparación de medias utilizando el ensayo Tukey. Estos valores se analizaron a través del software SAS 9.3 con un nivel de significancia $p<0,05$.

Tabla 2: Condiciones de temperatura y recubrimiento estudiados

\begin{tabular}{ll}
\hline Almacenamiento a $5^{\circ} \mathrm{C} \pm 2$ y $\mathrm{HR}$ de $85 \pm 2 \%$ & Almacenamiento a $15^{\circ} \mathrm{C} \pm 2$ y HR de $85 \pm 2 \%$ \\
\hline Formulación $1:\left(\mathrm{F} 15^{\circ} \mathrm{C}\right)$ & Formulación $1:\left(\mathrm{F} 115^{\circ} \mathrm{C}\right)$ \\
Formulación 2: $\left(\mathrm{F} 25^{\circ} \mathrm{C}\right)$ & Formulación $2:\left(\mathrm{F} 215^{\circ} \mathrm{C}\right)$ \\
Sin recubrimiento: Control $5^{\circ} \mathrm{C}$ & Sin recubrimiento: Control $15^{\circ} \mathrm{C}$ \\
\hline
\end{tabular}

\section{RESULTADOS Y DISCUSIÓN}

Para mayor claridad, los resultados son presentados en cuatro secciones: análisis sensorial, color, resistencia a la penetración, y análisis microbiológico.

\section{Análisis sensorial}

Los trozos de mango almacenados a la menor temperatura, Control $5^{\circ} \mathrm{C}$ y $\mathrm{F} 15^{\circ} \mathrm{C}$ presentaron las mejores notas sensoriales en cuanto a apariencia y sabor, con un nota promedio de 6,0 hasta el día 14 de la evaluación y sin diferencia estadística significativa $(p<0,05)$. Es decir sensoriamente hablando la presencia de ácidos grasos y almidón no presentó una diferencia en el sabor y la apariencia de los trozos de mango comparados con lo trozos control. En todos los demás tratamientos las menores notas sensoriales tanto de apariencia como de sabor fueron inferiores a 5 después del día 12 de almacenamiento. En los trozos de mango sin recubrimiento y en los recubiertos con la formulación F2 se observó un oscurecimiento notorio a partir del día 6 de almacenamiento, en los primeros seguramente debido al oscurecimiento enzimático con la presencia de oxígeno y en los recubiertos se cree que fue debido al elevado contenido de aceite de aguacate lo cual provocó en menos de una semana la oxidación de lípidos afectando su coloración y sabor. La cantidad de aceite de aguacate adicionada en la formulación F2 es el doble en comparación con la utilizada en la formulación F1. La aplicación previa de los antioxidantes ácido cítrico y ácido ascórbico evitó el oscurecimiento enzimático prematuro en los tratamientos Control $5^{\circ} \mathrm{C}$ y $\mathrm{F} 15^{\circ} \mathrm{C}$. La aplicación de recubrimientos crea una barrera semipermeable a gases similar a una atmosfera modificada lo que reduce la velocidad de respiración, la acción enzimática y la deshidratación de los productos (Chiumarelli et al., 2011).

En este trabajo se considera que hubo un conjunto de factores que evitaron el oscurecimiento enzimático prematuro de los trozos de mango como el uso del empaque al originar una atmosfera modificada baja en oxígeno (He y Luo, 2007), el propio recubrimiento comestible por su efecto barrera al contacto del oxígeno (Rojas-Graü et al., 2009), al uso previo al corte de ácido cítrico y ácido ascórbico (Robles-Sánchez, et al., 2013), el propio aceite de aguacate al actuar como agente reductor y atrapador de oxigeno (Silva-Weiss, et al., 2013) y la baja temperatura de almacenamiento que disminuye la actividad de las enzimas responsables (Guerrero-Eraso, 2009).

El sabor es una variable relacionado con el olor, por lo tanto, si el olor es desagradable el sabor se tornará desagradable. Las muestras control almacenadas a $5^{\circ} \mathrm{C} \pm 2$, presentaron un buen sabor 7,2 puntos, ya que el olor era característico de una fruta en fresco, en cambio las muestras que contenían recubrimiento F2 a $15^{\circ} \mathrm{C} \pm 2$, el sabor no fue agradable siendo esta la que presenta las concentraciones de los componentes lipídicos más altas. Realizando una comparación entre las muestras que contenían recubrimientos y la muestra control, los recubrimientos F1 y F2 no cumplieron con la característica de conservar las características de sabor del mango. Por otro lado la degradación de la calidad sensorial evaluada en la apariencia y sabor observada en los trozos de mango $\mathrm{F} 25^{\circ} \mathrm{C}$ y $\mathrm{F} 21^{\circ} \mathrm{C}$ puede ser atribuida a la mayor presencia de ácidos 
grasos monoinsaturados como el ácido oleico del aceite de aguacate (Silva-Weiss et al., 2013), contribuyendo a la rápida autooxidación originando oscurecimiento en el productos, aromas desagradables y sabor rancio (Azeredo, 2004).

El envase PET hermético crea una condición de atmósfera modificada contribuyendo de forma positiva a la conservación de los atributos de calidad como lo afirman Dussán-Sarria et al. (2014) y Singh et al. (2007). De igual forma la menor temperatura de almacenamiento reafirma que el uso del frio tiene efectos positivos en la conservación de frutas y hortalizas ya que disminuye la tasa respiratoria, la acción enzimática y por ende desacelera el proceso de maduración del producto (Ramaswamy, 2015).

\section{Color}

En la Figura 1 se observa que los valores de luminosidad $L^{*}$ disminuyeron a través del tiempo en todos los 6 tratamientos, siendo el tratamiento control $5^{\circ} \mathrm{C}$, control $15^{\circ} \mathrm{C}$ y $\mathrm{F}^{\prime} 15^{\circ} \mathrm{C}$ los que presentaron los valores más bajos de L* (Figura 1a y Figura $1 b$ ).
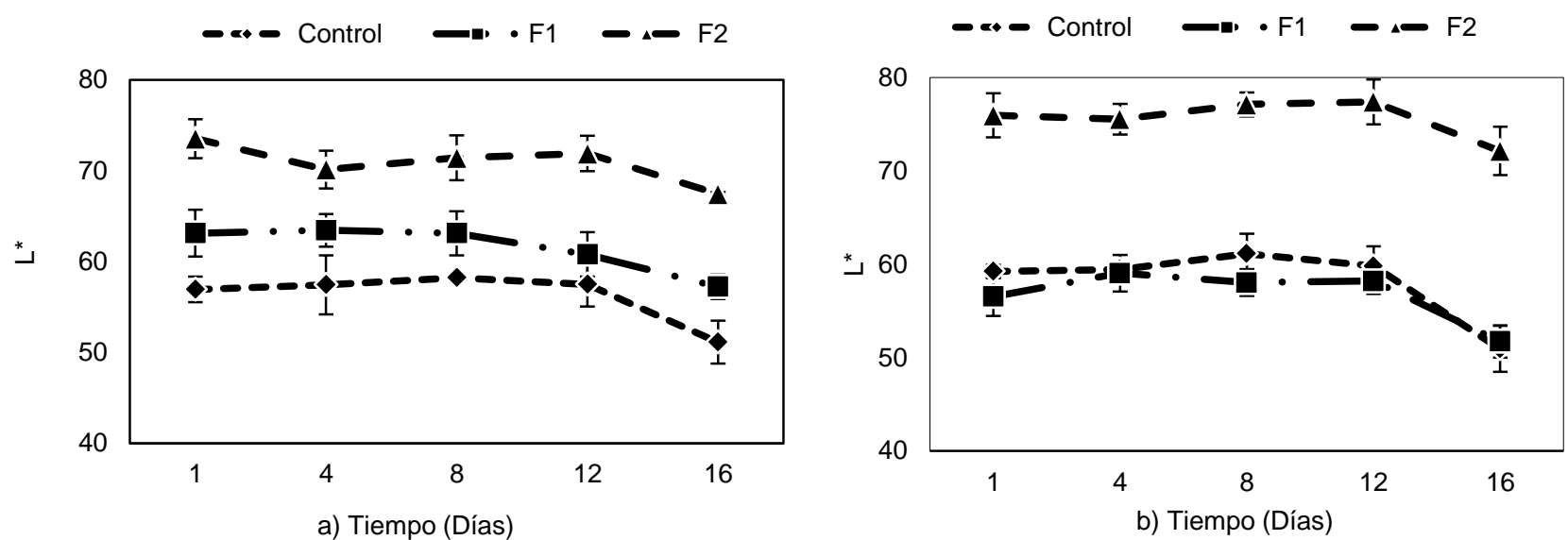

Fig. 1: Cambios en la luminosidad $\left(\mathrm{L}^{*}\right)$ de mango MP almacenado bajo refrigeración. a) $5 \pm 2^{\circ} \mathrm{C}$ y $80 \pm 2 \%$ de HR. b) $15 \pm 2^{\circ} \mathrm{C}$ y $80 \pm 2 \%$ de HR

A pesar de la inmersión del fruto en ácido cítrico como antioxidante los tratamientos control perdieron luminosidad hasta el día 16. Las muestras con la formulación F2, a las dos temperaturas, presentaron los mayores valores de luminosidad seguido de los trozos de mango con la formulación F1 y menores valores en los controles sin recubrimiento. Robles et al. (2013), encontraron que en cubos de mango de la variedad Kent con recubrimiento comestible basado en alginato solo perdieron $2,5 \%$ de su valor inicial de $L^{*}$ en comparación con una pérdida del $7 \%$ en las muestras sin recubrimiento. Puede ser que exista mayor grado de reflectancia de la luz captada por el colorímetro en productos con mayor contenido de ácidos grasos como la formulación F2. Revilla y Vivar (2005) encontraron que embutidos cárnicos con mayor contenido de ácidos grasos originan mayores valores de $L^{*}$. En todos los tratamientos los valores de $C^{*}$ disminuyeron debido a la disminución de los valores de $b^{*}$ y a la perdida de coloración amarilla.
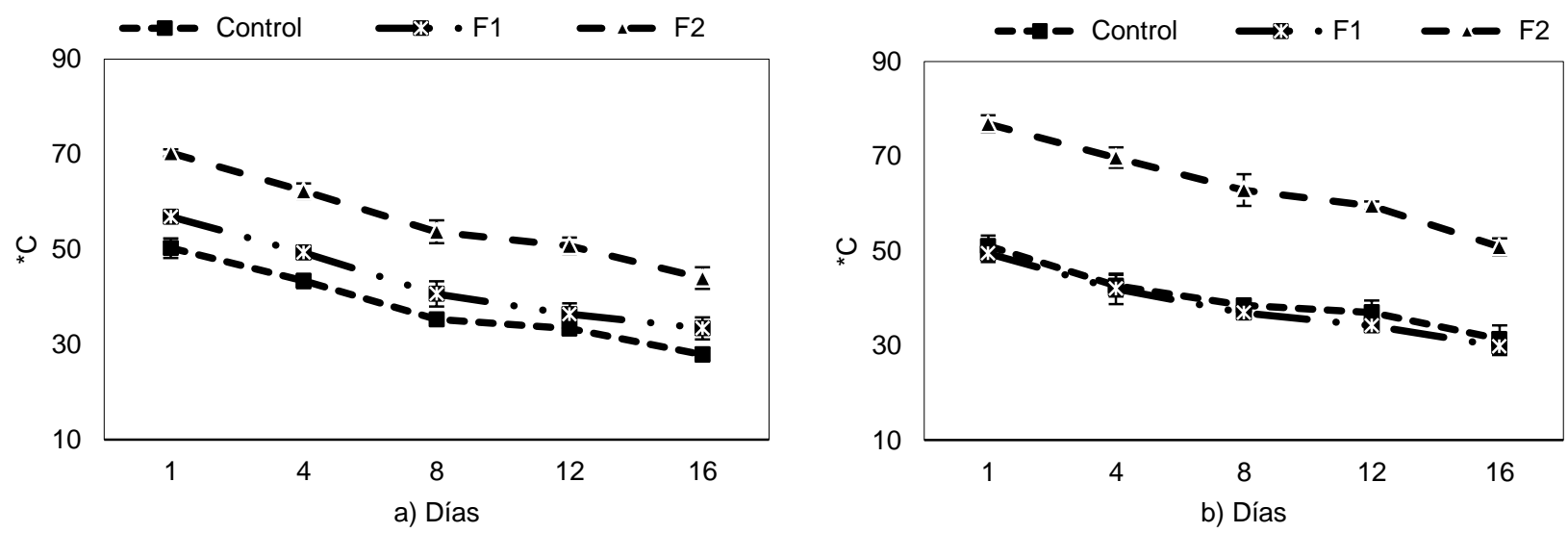

Fig. 2: Cambios en la cromaticidad $\left({ }^{*} \mathrm{C}\right)$ de mango MP almacenado bajo refrigeración. a) $5 \pm 2{ }^{\circ} \mathrm{C}$ y $80 \pm 2 \%$ de HR. b) $15 \pm 2^{\circ} \mathrm{C}$ y $80 \pm 2 \%$ de HR. 
Los frutos con la formulación F2 presentaron los mayores valores $C^{*}$ seguido del tratamiento $\mathrm{F} 1$ a ambas temperaturas de almacenamiento (Figura 2), mientras que los valores del tono $\left(\mathrm{H}^{*}\right)$ en todos los tratamientos se mantuvo constante, siendo los tratamientos $\mathrm{F} 1$ de mayor $\mathrm{H}^{*}$ y los controles de menor $\mathrm{H}^{*}$, lo que evidencia mayor oscurecimiento en los tratamientos F2 a ambas temperaturas (Figura 3). Los valores de Croma $\left(\mathrm{C}^{*}\right)$ y Tono $\left(\mathrm{H}^{*}\right)$ son importantes ya que consideran las variaciones en las coordenadas $a^{*}$ y $b^{*}$ abarcando el espectro de color del mango hasta su completa maduración con color amarillo intenso. La coordenada $\mathrm{H}^{\star}$ representa el color verdadero, eficaz para la visualización de la apariencia del color de los productos alimenticios (RoblesSánchez et al., 2013).
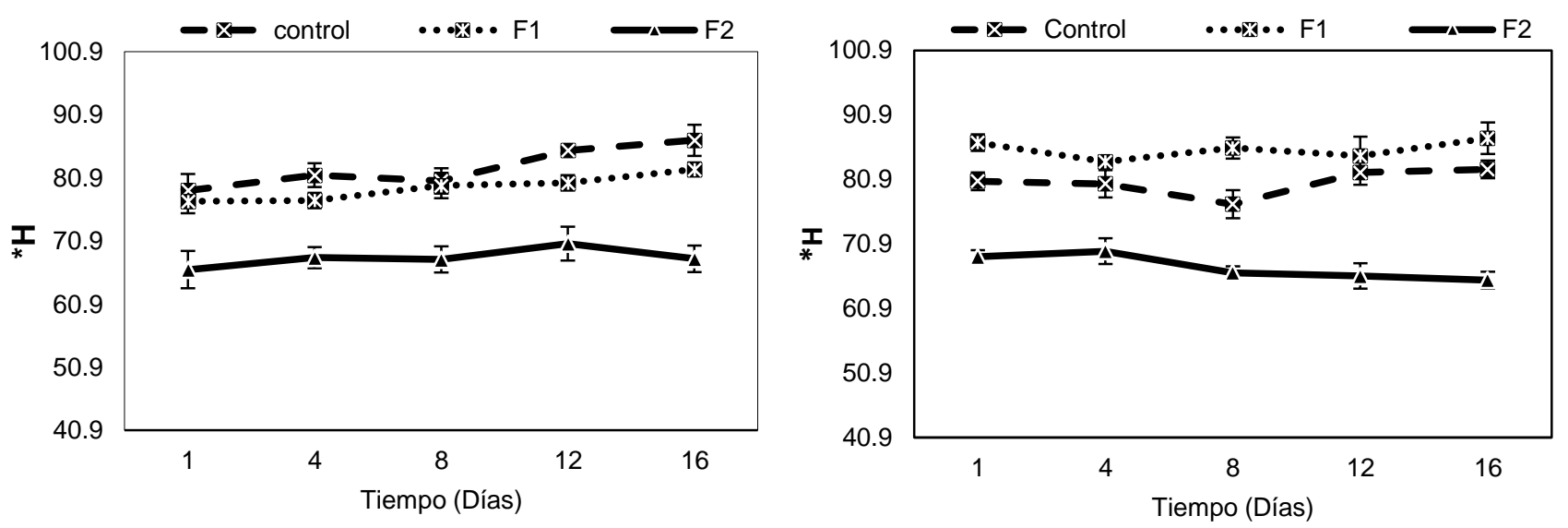

Fig. 3: Cambios en la tonalidad $\left({ }^{*} \mathrm{H}\right)$ de mango MP almacenado bajo refrigeración. a) $5 \pm 2^{\circ} \mathrm{C}$ y $80 \pm 2 \%$ de HR. b) $15 \pm 2^{\circ} \mathrm{C}$ y $80 \pm 2 \%$ de HR.

\section{Resistencia a la penetración}

En la Figura 4 se observa la perdida de resistencia a la penetración en los pedazos de mango durante el almacenamiento. Existe una relación inversamente proporcional entre la resistencia a la penetración y el tiempo de almacenamiento. En general los valores se comportaron de forma similar con los obtenido por Dussán-Sarria et al. (2014), variando en media de 2,7 kgf hasta 1,5 kgf en el día 16 del almacenamiento. La fruta cortada es muy perecedera debido a que son modificadas sus propiedades fisicoquímicas por los trastornos metabólicos que se producen por el corte (Kader, 2008), adicional a esto, este tipo procesamiento genera oscurecimiento y disminución de la firmeza (Chiumarelli et al., 2011), además el tratamiento previo de inmersión del fruto en ácido cítrico, ácido ascórbico y la adición de calcio, presenta buenos resultados al mantener la firmeza del mango 'Tommy Atkins' cortado (Siddiq et al., 2013). Estadísticamente se encontraron diferencias significativas en los tratamientos aplicados, así como entre los días de los tratamientos $(p<0.05)$.
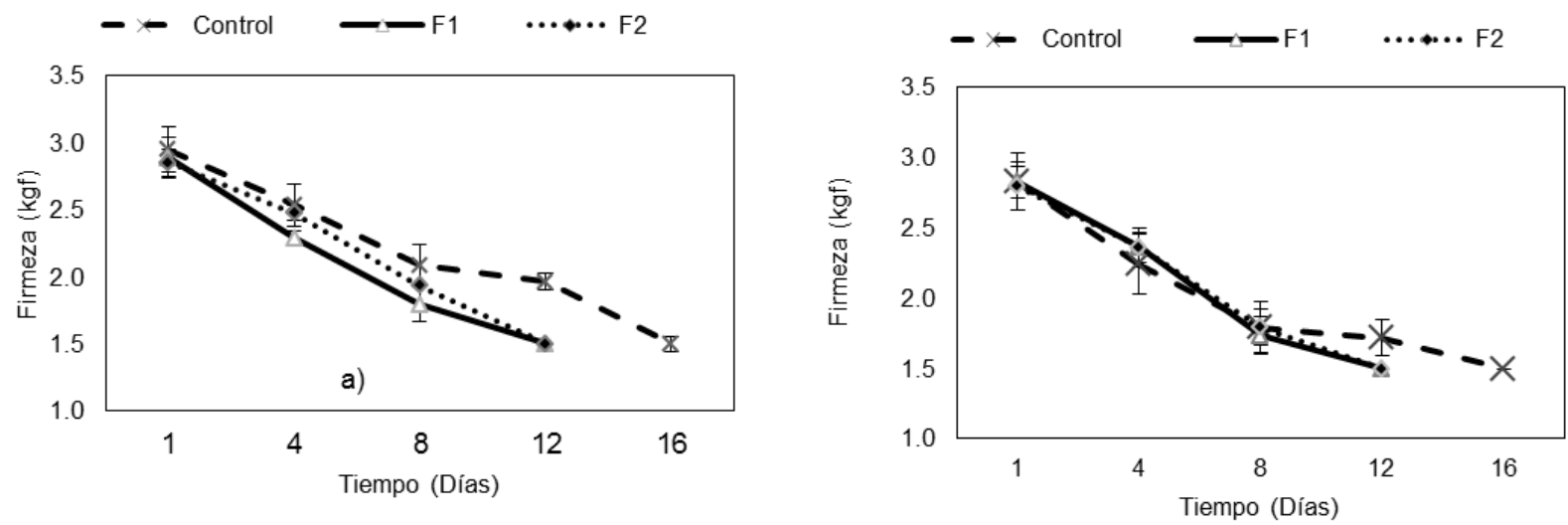

Fig. 4: Cambios en los valores de firmeza de mango MP almacenado bajo refrigeración. a) $5 \pm 2^{\circ} \mathrm{C}$ y $80 \pm 2 \%$ de $\mathrm{HR}$. b) $15 \pm 2^{\circ} \mathrm{C}$ y $80 \pm 2 \%$ de HR.

Los datos obtenidos durante la experimentación en el análisis sensorial y físico realizado, evidencian que para las muestras a $15^{\circ} \mathrm{C} \pm 2$ al final de experimento fueron las que tuvieron las pérdidas de peso más aceleradas presentando valores iniciales para F1 y F2 de 2,83 y 2,79 Kgf respectivamente y a los 12 días de 1,5 Kgf ambas. Este hecho evidencia que a pesar de que las formulaciones evaluadas presentaban diferentes concentraciones de ácido esteárico y cera de carnauba, los cuales, en un principio actuarían como protectores a la pérdida de agua o peso de los frutos, la condición de diferente temperatura de almacenamiento es la que 
realmente incide en la pérdida de peso. Los trozos de mango que presentaron mejor estabilidad durante el almacenamiento es el control bajo refrigeración a $5^{\circ} \mathrm{C} \pm 2$ y $80 \pm 2 \%$ de HR. El valor de la firmeza inicial de la muestras era adecuada al momento del corte, pero durante la refrigeración se provocó una pérdida de agua en los cubos de mango, que se manifestó con pérdida de firmeza. Análisis realizados por Souza et al. (2006), reportan un efecto negativo en la firmeza de rebanadas de mango 'Kensington' tratadas con calcio y ácido cítrico, según estos autores aunque el modo de acción de este proceso es desconocido lo que si pueden establecer es que la combinación de ácido cítrico con calcio duplica la pérdida de peso y por ende disminuye la firmeza. No se observó un efecto del almidón de yuca en la resistencia estructural del recubrimiento pues los trozos de mago control al final del almacenamiento exhibieron la misma resistencia a la penetración que los trozos de mango con recubrimiento a base de almidón.

\section{Análisis Microbiológico}

Al final del periodo de almacenamiento, los trozos de mango almacenados a $15 \pm 2^{\circ} \mathrm{C}$ presentaron valores superiores en coliformes totales y levaduras de acuerdo a los valores considerados tolerables según el INVIMA (2013), por otro lado, todos los tratamientos almacenados a $5 \pm 2^{\circ} \mathrm{C}$ presentaron valores inferiores a los mínimos tolerables en lo que respecta a coliformes totales, coliformes fecales, salmonella, mohos y levaduras. El corte realizado a los mangos crea mayor área superficial, disponibilidad de nutrientes y agua a los microorganismos pudiendo provocar una aceleración en el crecimiento microbiano, de igual forma las condiciones de temperaturas de refrigeración superiores a $5^{\circ} \mathrm{C}$ crean mejores condiciones de proliferación de microorganismos en los frutos cortados (Kader, 2002).

\section{CONCLUSIONES}

Los trozos de mago variedad Tommy Atkins con previa aplicación de ácido ascórbico $1 \% \mathrm{v} / \mathrm{v}$, ácido cítrico $1 \% \mathrm{v} / \mathrm{v}$ y $\mathrm{CaCl}_{2} 1 \% \mathrm{v} / \mathrm{v}$, posterior aplicación de recubrimiento comestible preparado con aceite de aguacate $(1,2 \% \mathrm{v} / \mathrm{v})$, almacenados a $5^{\circ} \mathrm{C} \pm 2$ y $80 \pm 2 \%$ de HR en empaques PET es la condición más adecuada en su conservación. Cuando es utilizada una proporción de aceite de aguacate en el recubrimiento comestible superior a $1,2 \% \mathrm{v} / \mathrm{v}$ se afectan negativamente los atributos sensoriales y físicos en detrimento de la calidad de los trozos de mango.

\section{REFERENCIAS}

Anzaldúa-Morales, A. La evaluación sensorial de los alimentos en la teoría y la práctica, Zaragoza, Acribia SA, 198 p. (1994)

Azeredo, H.M.C. de (Ed.). Fundamentos de estabilidade de alimentos, Fortaleza, Brasil, Embrapa Agroindústria Tropical, 195 p. (2004)

Báez, R., Bringas, E., Mendoza, A. M., González, A.G., y Ojeda-Conteras, A.J. Recubrimientos de tratamientos especiales en frutos de mango tratados hidrotérmicamente, $2^{\circ}$ Congreso lberoamericano de Tecnología Post-cosecha y Agro-exportaciones, p. 71-74 (2000)

Barbagallo, R. N., Chisari, M. y Spagna, G. Enzymatic browning and softening in vegetable crops: Studies and experiences, Italian J. Food Sci., 21(1) 3-16 (2009)

Chien, P., Sheu F. y Yang F. Effects of Edible Chitosan Coating on Quality and Shelf Life of Sliced Mango Fruit, Journal of Food Engineering, 78 (1), 225-229 (2007)

Chiumarelli, M., Cristhiane, C.F., Sarantópoulos, C. y Hubinger, M. Fresh Cut 'Tommy Atkins' Mango Pretreated with Citric Acid and Coated with Cassava (Manihot Esculenta Crantz) Starch or Sodium Alginate, Innovative Food Science \& Emerging Technologies, 12 (3), 381-387 (2011)

Djioua, T., Charles, F., Lopez-Lauri, F., Filgueiras, H., Coudret A., Freire M., Ducamp-Collin, M. y Sallanon $\mathrm{H}$. Improving the Storage of Minimally Processed Mangoes (Mangifera Indica L.) by Hot Water Treatments, Postharvest Biology and Technology, 52 (2) 221-226 (2009)

Djioua, T., Charles, F., Freire, M, Filgueiras, H., Ducamp-Collin, M. y Sallanon, H. Combined Effects of Postharvest Heat Treatment and Chitosan Coating on Quality of Fresh-cut Mangoes (Mangifera Indica L.). International Journal of Food Science \& Technology, 45 (4), 849-855 (2010)

Dussán-Sarria, S., Torres-León, C., y Hleap-Zapata, J.I. Efecto de un Recubrimiento Comestible y de Diferentes Empaques durante el Almacenamiento Refrigerado de Mango Tommy Atkins Mínimamente Procesado, Información Tecnológica, 25(4), 123-130 (2014) 
Estrada-Mesa, E. M., Padilla-Reyes, F., Márquez-Cardozo, C. J. Efecto de recubrimientos protectores sobre la calidad del mango (Mangifera indica L.) en poscosecha, Rev. U.D.C.A Act. \& Div. Cient., 18(1), 181-188 (2015)

Guerrero-Eraso, C. A. Inhibición de la actividad enzimática de la polifenol oxidasa extraída del banano (Cavendish valery) mediante sistemas bifásicos acuosos con isoespintanol y ácido ascórbico. Tesis de Maestría en Ciencia y Tecnología de Alimentos. Universidad Nacional de Colombia. 91p. (2009)

He, Q., Luo, Y. Enzymatic browning and its control in fresh-cut produce, Stewart Postharvest Review, 6, 3 (2007)

IFPA - International Fresh-cut Produce Association. 'Fresh-cut Produce Fuels An America On-the-go', IFPA, 2004, http://www.fresh-cuts.org. Acceso: 12 de noviembre (2016)

INVIMA - Instituto Nacional de Vigilancia de Medicamentos y Alimentos. Ministerio de Protección Social. Colombia. Res. 3929. 29p. (2013)

Kader, A.A. Post-harvest technology of horticultural crops. Oakland: University of California, Division of Agriculture and Natural ResourcesPublication, 3311, 535 p. (2002)

Kader, A. A., Fresh-cut Mangos as a Value-added Product (Literature Review and Interviews), Orlando, FL, USA (2008)

Kechichian, V., Ditchfield, C., Veiga-Santos, P., Tadini, C. Natural antimicrobial ingredients incorporated in biodegradable fi Ims based on cassava starch. LWT - Food Science and Technology, 43(7) 1088-1094 (2010)

Laurila, E., Kervinen, R. y Ahvnainen, R. The inhibition of enzymatic browning in minimally processed vegetable and fruits. Posth. News and Information, 9(4), 53-65 (1998)

Liu, F., Qin, B., He, L., Song, R. Novel starch/chitosan blending membrane: antibacterial, permeable and mechanical properties, Carbohydrate Polymers, 78, 146 - 150 (2009)

Pérez-Gago, M. N., Jong-Whan, R. Edible Coating and Film Materials: Lipid Bilayers and Lipid Emulsions, Chapter 13, In: Han, J. H. Innovations in Food Packaging, $2^{\text {nd }}$ Ed., Elsevier Ltda., 325-350 (2014)

Ramaswamy, H. S. Post-harvest Technologies of Fruits \& Vegetables, Ed., DEStech Publications, Inc. Pennsylvania-USA, 311p. (2015)

Revilla, I., y Vivar, A.M. The effect of different paprika types on the ripening process and quality of dry sausages, International Journal of Food Science and Technology, 40 (4), 411-417 (2005)

Robles-Sánchez, R. M., Rojas-Graü, M. A., Odriozola-Serrano, I., González-Aguilar, G., Martin-Belloso, O. Influence of Alginate-Based Edible Coating as Carrier of Antibrowning Agents on Bioactive Compounds and Antioxidant Activity in Fresh-Cut Kent Mangoes. LWT - Food Science and Technology, London, 50 (1) $240-246$ (2013)

Rojas-Graü, M. A., Soliva-Fortuny, R., y Martin-Belloso, O. Edible coatings to incorporate active ingredients to fresh-cut fruits: A review, Trends in Food Science and Technology, 20 (10) 438-447 (2009)

Siddiq, M., Sogi, D., K. Dolan. Antioxidant properties, total phenolics, and quality of fresh-cut "Tommy Atkins" mangoes as affected by different pre-treatments, Food Science and Technology, 53, 156-162 (2013)

Silva-Weiss, A., Ihl, M., Sobral, P. J. A., Gómez-Guillén, M.C., Bifani, V. Natural Additives in Bioactive Edible Films and Coatings: Functionality and Applications in Foods, Food Eng. Rev. 5, 200 (2013)

Singh, Z., Singh, R., Sane, V. y Nath, P. Mango - Postharvest Biology and Biotechnology, Critical Reviews in Plant Sciences, 32(4), 217-236 (2013)

Sothornvit, R. y Rodsamran, P. Effect of a Mango Film on Quality of Whole and Minimally Processed Mangoes. Postharvest Biology and Technology, 47 (3), 407-415 (2008)

Souza, B. S., O'Hare, T. J., Durigan, J. F. y Souza, P. S. Impact of Atmosphere, Organic Acids, and Calcium on Quality of Fresh-cut 'Kensington' Mango, Postharvest Biology and Technology, 42 (2), 161-167 (2006)

Tharanathan, R. N., Yashoda, H. M., y Prabha, T. N. Mango (Mangifera indica L.), "The King of Fruits" - An overiew, Food Reviews International, 22, 95-123 (2006) 